\title{
EXPERIMENTAL STUDY OF THE CONVECTIVE HEAT TRANSFER FROM TUBES WITH VARIOUS CROSS- SECTION
}

\author{
Muhammad Chrissandy Fahzy, Yonas Paskah Pardede, Danel Reka Yesa, \\ MOHD RADEN BAHI, KAPRAWI SAHIM* \\ Department of Mechanical Engineering, Faculty of Engineering, Universitas Sriwijaya, Indralaya, \\ Indonesia \\ *Corresponding author: kaprawi@unsri.ac.id
}

(Received: 28 January 2022; Accepted: 19 February 2022; Published on-line: 01 July 2022)

\begin{abstract}
In heat transfer engineering, most of the tubes of the circular cross-section are used in the heat exchanger application, but the possibilities of using tubes other than circular type are available to enhance the rate of heat transfer. This experimental study presents the heat transfer rate performance of the tubes' difference cross-section; circular, elliptic, square, and triangle tubes. The tubes were heated at uniform heat flux at the outside surface, and the water flowed inside the tubes. The important parameters were measured during the experimentation. The results show that the heat transfer varies with the cross-section type. The triangle and elliptical tube give a higher heat transfer rate than other cross-sections.
\end{abstract}

KEYWORDS: Convective heat transfer, Tubes, heat transfer performance, entry length, constant heat flux.

\section{INTRODUCTION}

The heat exchanger is the apparatus for transferring the heat from one medium to another. This apparatus commonly consists of the tubes that have the function to transfer from fluid flowing inside and the flow flowing at the outside of the tubes. Three types of ducts: circular, rectangle type, and triangle ducts, were tested by Saleh et al. [1] to know the heat transfer performance. The ducts were heated at the constant heat flux with an electric current outside the ducts. The results show that the triangle duct has the highest heat transfer, and the rectangular duct gives better performance than the circular type. Similarly, Rasheed et al. [2] carried out similar work, which studied circular, rectangular and triangle cylinders in porous media forced heat transfer convection. They showed that the triangle cylinder has the highest heat transfer value than the square and circular cylinder.

Sutthivirode and Suparos [3] explored the forced convection heat transfer with air flowing in a different form of the duct cross-section, but the cross-section of all ducts are identical. The circular, square, and triangle ducts were investigated, and the constant heat flux assumption was applied for each experimentally tested case. The circular duct provided the highest performance compared to the rectangular and triangle duct. Heat transfer for constant wall temperature, Bisht et al. [4] gives numerically the study of comparison of the heat transfer of the water flowing in the circular and rectangular tubes used in the heat exchanger. They presented that the rectangular tubes give the significant difference in which the rectangular tubes are higher than that of the circular tubes. Dang et al. [5] compared the thermal performance of the circular tube with coil and without coil in the tube. The thermal performance is enhanced for tubes with the inserted coil in the circular tube. The square cylinder was 
explored experimentally for low Reynolds number and isothermal condition by Elsherbiny et al. [6].

The same study for low Reynolds numbers was carried out by Heris et al. [7] using nanofluid, which compared the heat transfer performance from the square and the triangle cross-sectional ducts. In this case, the square duct gives better results than the triangle duct. For high Reynolds number, the thermal performances of the triangle duct were studied by Kumar et al. [8] . It can be explained that the optimum heat transfer from triangle cross-sectional duct takes place under axially uniform wall heat flux with peripheral uniform wall temperature boundary conditions compared to all other boundary conditions. It is also shown that the heat transfer varies linearly with the Reynolds number. Every non-circular duct can be represented by the aspect ratio of side length or major/minor axis of the duct. The effect of this parameter in the combined entry region of the duct determined the rate of heat transfer as Muzychka and Yovanovich [9] presented it.

In a fully developed flow, the thermal performance function of Brinkman number of the equilateral triangle cross-section duct was conducted analytically by Norouzi et al. [10]. The fully developed laminar flow regime in the triangle duct's annulus channels, Rao et al. [11] explored numerically and experimentally the heat transfer characteristics, where they investigated the effect of viscous dissipation. Then, in laminar fully developed flow region in the circular pipe was investigated analytically [11]. The study compared the heat transfer performance for constant heat flux and constant wall temperature. Yang et al. [12] explored the heat transfer characteristics of turbulent flow in elliptical tubes with some aspect ratio numerically. The optimum heat transfer rate occurs in elliptical tubes with the semi-major axis length is twice the semi-minor axis's length.

Taler [13] presents the heat transfer correlation for turbulent flow in circular tubes where the theoretical and the experimental studies were compared. Kaprawi et al. [14] experimented with the entry length of the triangle channel of nanofluid flow with constant heat flux to know the heat transfer performance. For the flow across the cylinders[15], Janjua et al. [15] explored the forced convection heat transfer from the cylinder with various cross-sections. Generally, the forced heat transfer from the cylinders, the Nusselt number increases with increasing Reynolds number. The literature reviews seem that the heat transfer performances from the tubes with variable cross-sections depend on some parameters. In this study, it is presented the performance of heat transfer from various cross-section with the same area of the cross-section. Water was flowed inside of the tubes and it absorbs the heat given by the cylinder wall at constant heat flux.

\section{METHODS}

This research used an experimental method and made the experimental apparatus for data collection. The test is conducted by varying the cross-section of the tube and the flow rate. The test tube is made of copper material with an inner diameter of $12,7 \mathrm{~mm}$ for circular tube, an aspect ratio of 1 with a height of $10 \mathrm{~mm}$ and a width of $10 \mathrm{~mm}$ for a square tube, a side length of $12,7 \mathrm{~mm}$ for a triangular tube, and an aspect ratio of 0,4 with a major axis $15 \mathrm{~mm}$ and minor axis $6 \mathrm{~mm}$ for the elliptical tube. All types of tubes used have a length of $1000 \mathrm{~mm}$ and a thickness of $0,5 \mathrm{~mm}$. The tube is wrapped with an electric coil made of nichrome a size of 26 AWG ( $\phi=0,405 \mathrm{~mm})$ with a pitch distance of $15 \mathrm{~mm}$. Then between the surface of the tube and the electric coil, asbestos-based insulation is wrapped with a width of $8 \mathrm{~mm}$ therefore during heating there is no short circuit. Insulation is applied to cover the electric coil and the surface of the tube as a whole. Therefore, heat is not lost to the atmosphere. The geometry of the cross-section is presented in Fig. 1. 

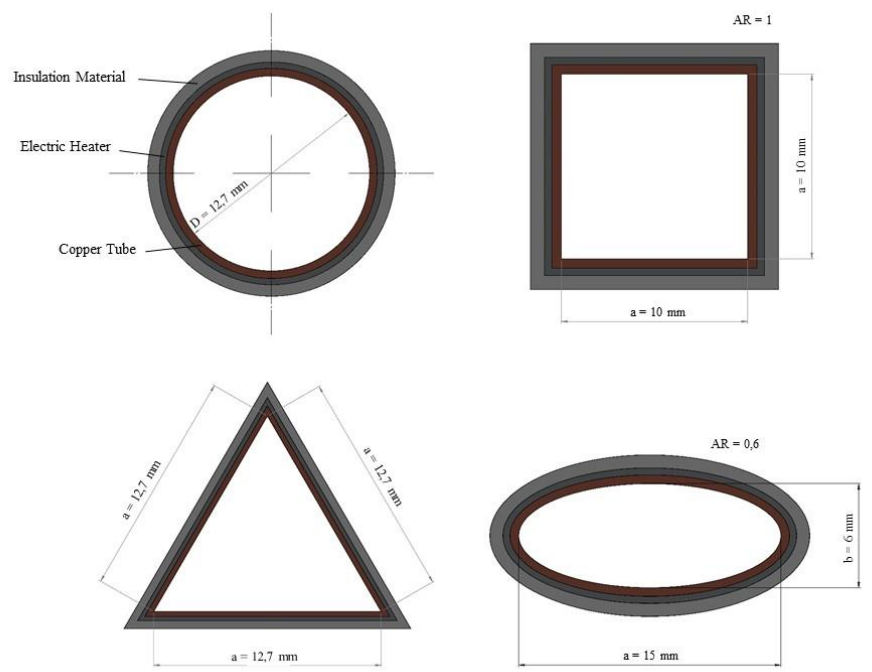

Fig. 1. Geometry of Cross-Section

The Schematic arrangement of the experimental apparatus in this study is shown in Fig. 2.

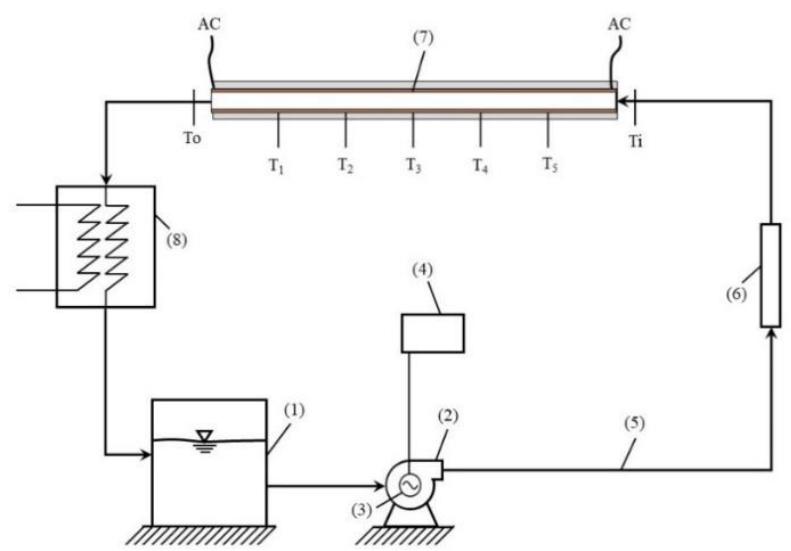

Fig. 2. The Schematic Arrangement of the Experimental Apparatus

After making the experimental apparatus, the following is a photo of the main element shown in Fig. 3.

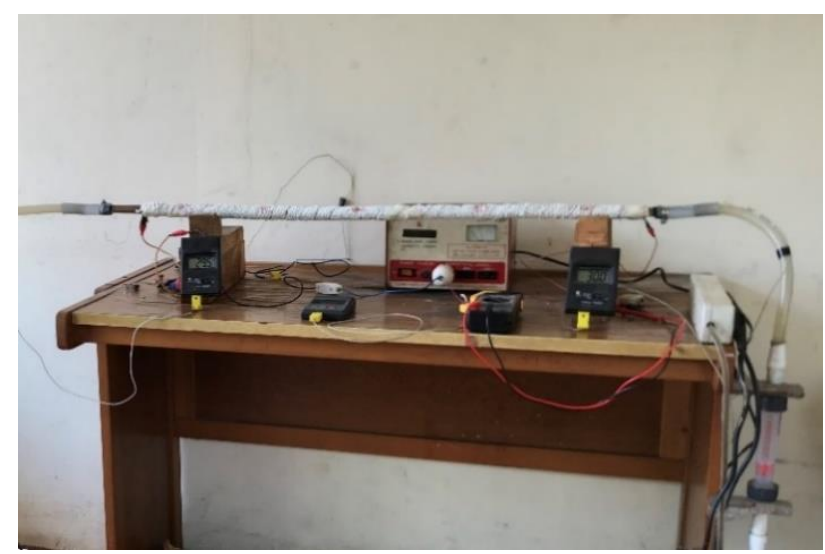

Fig. 3. Photo of Main Element 
The testing procedure carried out in this study begins with preparing and installing all the tools used in the test according to the schematic arrangement of the experimental apparatus. In this test, the water the working fluid is accommodated in the reservoir (1). From Fig. 2, water from the reservoir is pumped by a centrifugal pump (2) with a maximum capacity of $800 \mathrm{l} / \mathrm{h}$ to the test tube (7), which flows through a rubber hose (5) with a size of $1 / 2$ inch PVC type. The centrifugal pump is driven by a DC electric motor (3) connected to a voltage regulator (4) to regulate the flow rate. Before entering the test tube, the water passes through a floating type flowrate rotameter (6) with a maximum capacity of $160 \mathrm{l} / \mathrm{h}$, used to measure flow rate. A TM902C type-K digital thermocouple is also mounted on the tube inlet side.

Next, the water enters the $1000 \mathrm{~mm}$ test tube, wrapped in the copper tube around an electric coil and closed with insulation. The thermocouple is attached to the outer wall of the tube of 5 points, each point having the same distance of $16,5 \mathrm{~cm}$. The test tube is heated with AC voltage at 110 volts from the transformer connected to the electric coil. The heating process in the test tube causes the water that comes out of the test tube to pass through the thermocouple on the tube outlet side. Then the water passes through a cooling tower (8) spray type, which is used to return the water to the initial temperature of the test. Inside the cooling tower, a fan and hot water is distributed in the form of small sprays that spread in all horizontal directions. Then the cooled waterfalls into the basin and returns to the reservoir by gravity.

There are four variations of tube cross-section: circular tube, square tube, triangular tube, and elliptical tube for each flow rate variation from $20 \mathrm{l} / \mathrm{h}$ to $160 \mathrm{l} / \mathrm{h}$ with an increase of $10 \mathrm{l} / \mathrm{h}$. After the temperature tends to be stable, measurements of the voltage (V) and current (I) enter the heater first. Simultaneously, the water temperature at inlet (Ti), outlet (To), and surface/wall temperature of the outside of the tube $\left(T_{1}-T_{5}\right)$ was measured. This temperature measurement continues to be carried out until other variations of flow discharge.

Based on the test results data, analysis or calculation of the data is carried out using mathematical equations or relationships (1) to (13). The hydraulic diameter, $\mathrm{D}_{\mathrm{h}}(\mathrm{m})$, is calculated with the equation:

$$
D_{h}=\frac{4 A}{p}
$$

where $A$ is the cross-sectional area of the tube $\left(\mathrm{m}^{2}\right)$ and $\mathrm{p}$ is the wetted perimeter $(\mathrm{m})$. In a circular tube $D_{h}=D$. The average water temperature, $\operatorname{Trf}_{1}(K)$, is calculated by the equation:

$$
\operatorname{Tr} f_{1}=\frac{T i+T o}{2}
$$

and the difference in water temperature, $\Delta \mathrm{T}(\mathrm{K})$, is calculated by the equation:

$$
\Delta T=T o-T i
$$

$\mathrm{Ti}$ is the inlet water temperature $(\mathrm{K})$, and To is the outlet water temperature (K). Density $\left(\mathrm{kg} / \mathrm{m}^{3}\right)$; specific heat, $\mathrm{c}_{\mathrm{p}}\left(\mathrm{J} / \mathrm{kg} .{ }^{\circ} \mathrm{C}\right)$; dynamic viscosity, $(\mathrm{kg} / \mathrm{ms})$; and thermal conductivity, $\mathrm{k}$ $(\mathrm{W} / \mathrm{m} . \mathrm{K})$ in water is calculated by interpolating the average water temperature based on the table of properties of saturated water. The equation calculates kinematic viscosity, $\mathrm{v}(\mathrm{m} 2 / \mathrm{s})$ :

$$
v=\frac{\mu}{\rho}
$$

The equation calculates the average tube outer surface temperature, $\operatorname{Trs}_{2}(\mathrm{~K})$ :

$$
\operatorname{Tr}_{2}=\frac{T_{1}+T_{2}+T_{3}+T_{4}+T_{5}}{5}
$$

where $T_{1}$ to $T_{5}$ is the outer surface temperature of the tube $(\mathrm{K})$ at point 1 to point 5 . The equation calculates the flow velocity, $\mathrm{V}(\mathrm{m} / \mathrm{s})$ : 


$$
V=\frac{Q_{o}}{A}
$$

where $Q_{o}$ is the flow rate $\left(\mathrm{m}^{3} / \mathrm{s}\right)$. Reynolds number (The equation calculates ren:

$$
R e=\frac{V D_{h}}{v}
$$

The equation calculates the electric heating power, Qe (W):

$$
Q_{e}=I \times V
$$

where $\mathrm{I}$ is the current $(\mathrm{A})$ and $\mathrm{V}$ is the voltage $(\mathrm{V})$. The equation calculates the heat transfer rate, Qf $(\mathrm{J} / \mathrm{s})$ :

$$
Q_{f}=m c_{p} \Delta T=\rho V A c_{p} \Delta T
$$

where $\mathrm{m}$ is the mass flow rate $(\mathrm{kg} / \mathrm{s})$. The equation calculates loss temperature, Tloss $(\mathrm{K})$ :

$$
T_{\text {loss }}=\frac{Q_{f} \ln \left(r_{o h} / r_{i h}\right)}{2 \pi k_{\text {copper }} L}
$$

where $r_{\text {oh }}$ is the outer radius of the tube and $r_{i n}$ is the inner radius of the tube. $k_{\text {copper }}$ is the thermal conductivity of copper material $(\mathrm{W} / \mathrm{m} . \mathrm{K})$ obtained from the properties of the solid metals table, and $\mathrm{L}$ is the length of the tube $(\mathrm{m})$. The equation calculates the average inner surface temperature of the tube (Tis):

$$
T_{i s}=\operatorname{Trs}_{2}-T_{\text {loss }}
$$

The equation calculates the convective coefficient, $\mathrm{h}(\mathrm{W} / \mathrm{m} 2 . \mathrm{K})$ :

$$
h=\frac{Q_{f}}{\pi D_{h} L\left(T_{i s}-\operatorname{Tr} f_{1}\right)}
$$

Nusselt number, $\mathrm{Nu}$ is calculated by the equation:

$$
N u=\frac{h D_{h}}{k}
$$

\section{RESULT AND DISCUSSION}

From the analysis or calculation of data that has been carried out on the circular tube, square tube, triangular tube, and elliptical tube, the graph is shown in Fig. 4 to Fig. 5.

Based on Fig. 4, the elliptical tube has the largest water temperature difference compared to tubes with other cross-sections. They were then followed by a triangular tube and circular tube with a greater water temperature difference than a square tube. However, at a certain point (at the beginning of the flow), the square tube has a higher temperature value than the triangular tube. There is a very small difference in the value of the water temperature difference; therefore, the overall comparison of the water temperature difference from the circular tube and the triangular tube has a decreasing trend, and the value is relatively almost the same. The value of the water temperature difference indicates the increase in water temperature due to heating in the test tube. The magnitude of this temperature increase is influenced by the tube's outer radius, which is based on the thickness and hydraulic diameter of the tube. Tubes with a smaller hydraulic diameter have a smaller outer radius of the tube; therefore, the heat transfer will be faster. The graph also shows that the greater the Reynolds number, the lower the water temperature difference, both in elliptical, triangular, square, and circular tubes because a high Reynolds number has a large flow velocity. Therefore the contact time between the water and the tube surface becomes faster and causes the water temperature to decrease. When the Reynolds number is around 700 to 5000 , the maximum and minimum water temperature 
differences obtained for each variation of the tube cross-section are $13,1{ }^{\circ} \mathrm{C}$ and $3,4{ }^{\circ} \mathrm{C}$ for elliptical tube; $9,1{ }^{\circ} \mathrm{C}$ and $3,2{ }^{\circ} \mathrm{C}$ for triangular tube; $11,0{ }^{\circ} \mathrm{C}$ and $2,2{ }^{\circ} \mathrm{C}$ for square tube; and $12,8^{\circ} \mathrm{C}$ and $2,9^{\circ} \mathrm{C}$ for the circular tube.

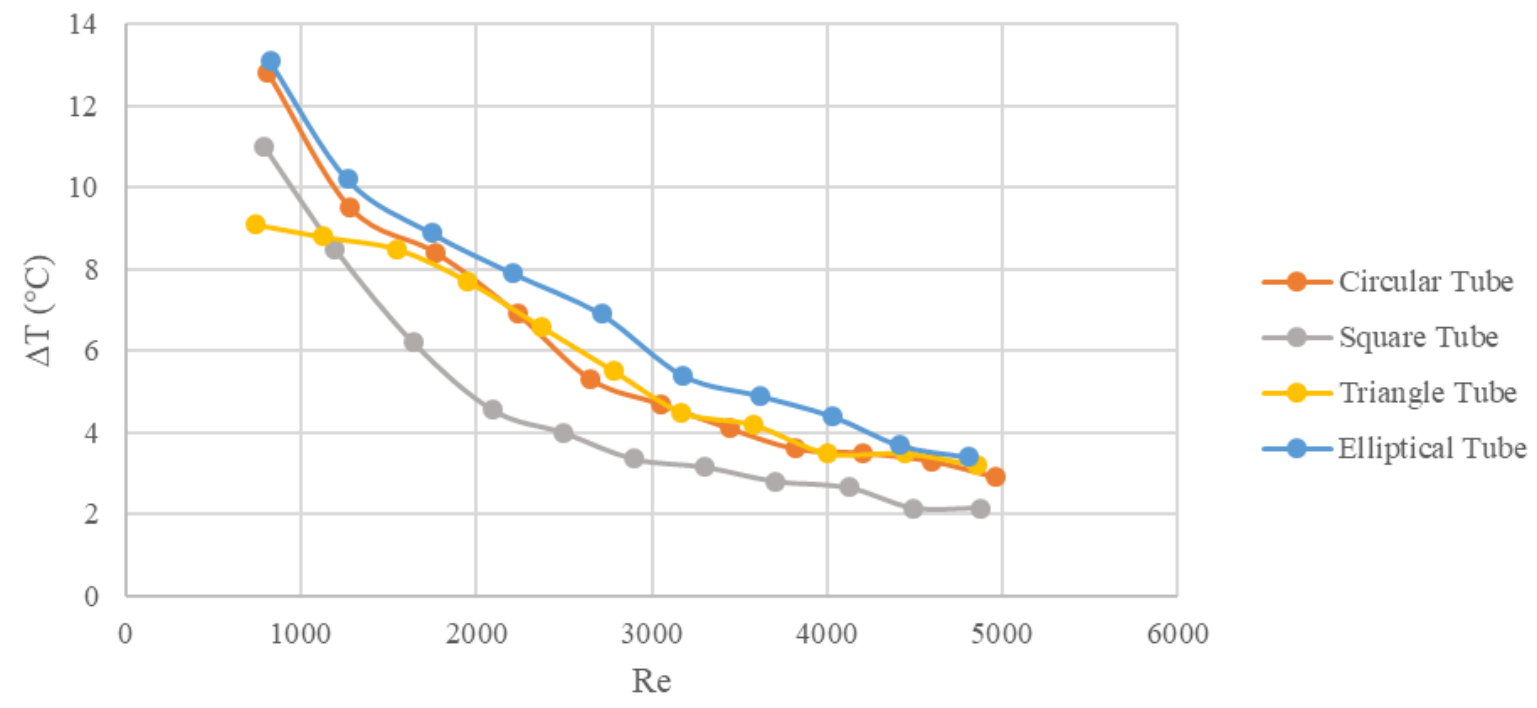

Fig. 4. Water Temperature Difference against Reynolds Number Graphic

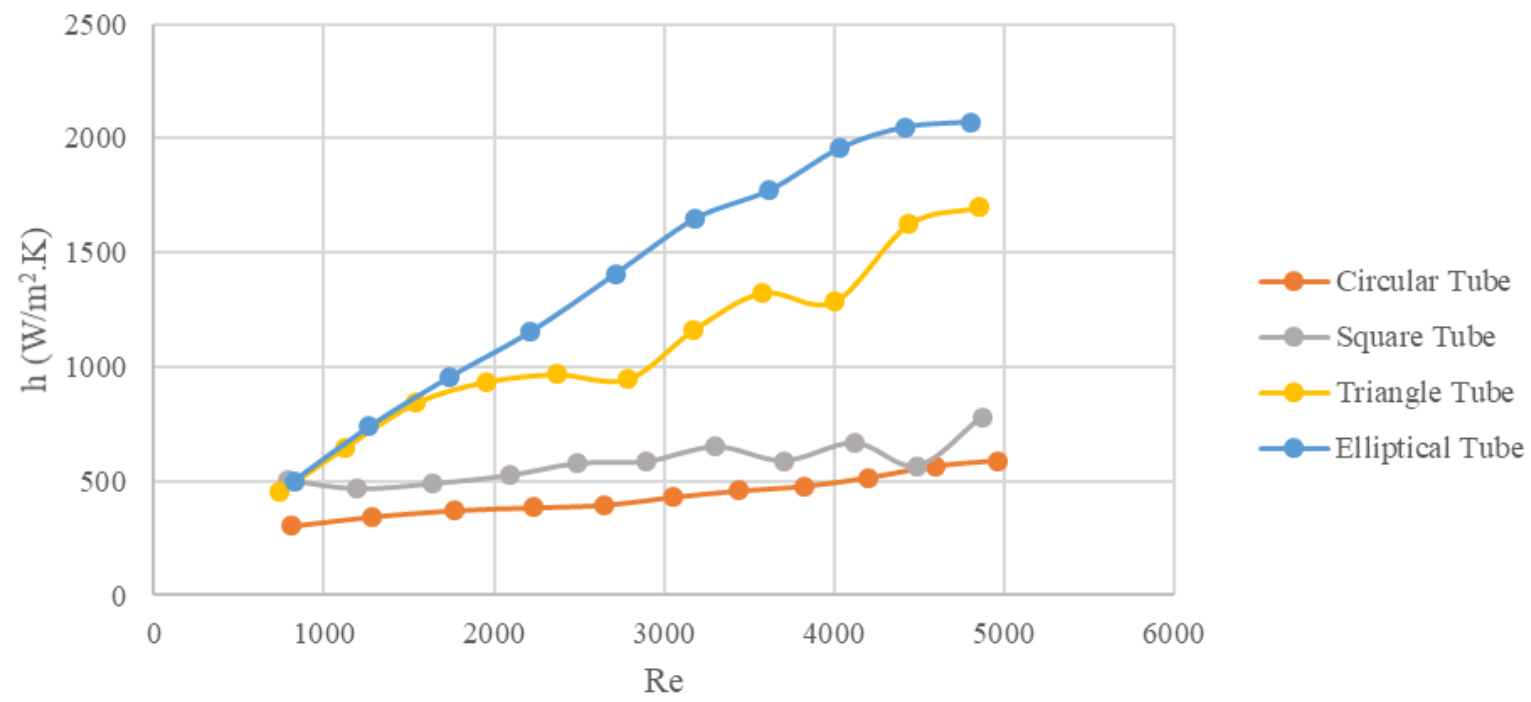

Fig. 5. Convective Coefficient against Reynolds Number Graphic

Based on Fig. 5, the a large difference in the value of the convective coefficient between the elliptical tube and the triangular tube with the square tube and circular tube. The elliptical tube has the highest convective coefficient compared to tubes with other cross-sections. Then sequentially, the triangular tube has a higher convective coefficient than the square tube, followed by the circular tube. The increase in the convective coefficient is influenced by the magnitude of the heat transfer rate and the temperature difference between the surface/wall of the tube and the temperature of the water. The value of the heat transfer rate is influenced by the magnitude of the mass flow rate. The larger the hydraulic diameter of the tube, the greater the cross-sectional area of the tube, therefore the mass flow rate will increase. Although the circular tube has a greater increase in water temperature than the square tube, the square tube 
has a higher convective coefficient value than the circular tube because the square tube has a larger mass flow rate. The greater the rate of heat transfer, the convective coefficient will increase. While the convective coefficient will decrease if the temperature difference between the surface of the tube and the water is getting bigger. The graph also shows that the greater the Reynolds number, the greater the convective coefficient for elliptical tubes, triangular tubes, square tubes, and circular tubes. This is also influenced by the value of the heat transfer rate, which increases as the flow velocity increases. The increase in the convective coefficient is low in laminar flow conditions and is more significant in turbulent flow conditions. When the Reynolds number is around 700 to 5000, the maximum and minimum convective coefficients obtained for each tube cross-section are $2069,035 \mathrm{~W} / \mathrm{m}^{2} . \mathrm{K}$ and $500,341 \mathrm{~W} / \mathrm{m}^{2} . \mathrm{K}$ for elliptical tube; $1699,549 \mathrm{~W} / \mathrm{m}^{2} . \mathrm{K}$ and $850,091 \mathrm{~W} / \mathrm{m}^{2} . \mathrm{K}$ for triangular tube; $779,099 \mathrm{~W} / \mathrm{m}^{2} . \mathrm{K}$ and $466,746 \mathrm{~W} / \mathrm{m}^{2} . \mathrm{K}$ for square tube; and $588,712 \mathrm{~W} / \mathrm{m}^{2} . \mathrm{K}$ and $305,609 \mathrm{~W} / \mathrm{m}^{2} . \mathrm{K}$ for circular tube.

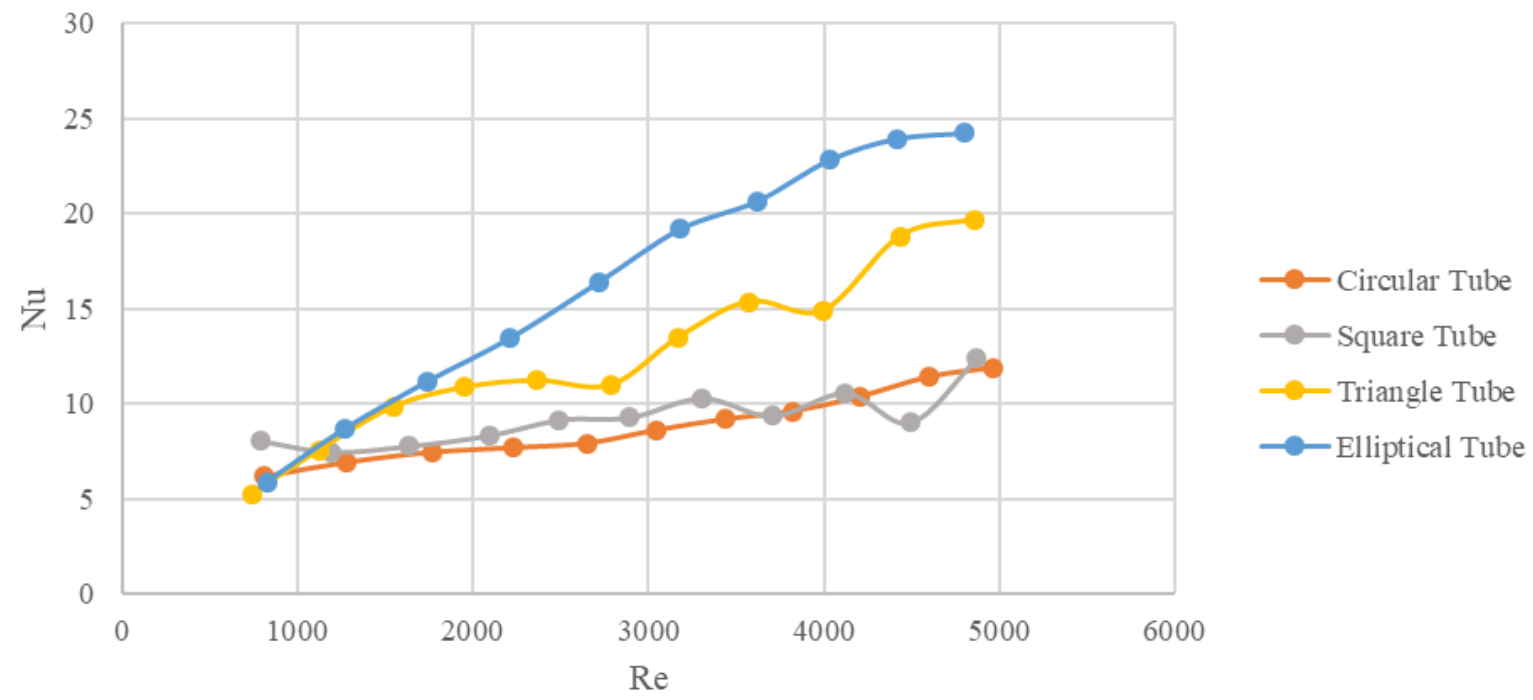

Fig. 6. Nusselt Number against Reynolds Number Graphic

Based on Fig. 6, the elliptical tube has the largest Nusselt number compared to the tube with other cross-sections. Then sequentially, the triangular tube has a Nusselt number greater than the square tube, followed by the circular tube. However, at a certain point towards the end of the flow, the square tube has a lower Nusselt number than the circular tube, then increases again and is larger than the circular tube at the end of the flow. The comparison of the Nusselt number values has an increasing trend that is relatively the same as the convective coefficient since the Nusselt number is influenced by the convective coefficient value and the hydraulic diameter of the tube. Increasing the convective coefficient and the hydraulic diameter of the tube, the greater the Nusselt number.

In contrast, The Nusselt number considers the thermal conductivity of the fluid due to the influence of heat transfer that occurs by conduction when the fluid layer does not move and the thermal conductivity of the tube material used. On the other hand, the value of the Nusselt number will be smaller as the thermal conductivity of the fluid and tube material increases. The graph also shows that the greater the Reynolds number, the Nusselt number will increase, both for elliptical tube, triangular tube, square tube, and circular tube. This is also influenced by the value of the heat transfer rate, which increases as the flow velocity increases. The increase in Nusselt number is low in laminar flow conditions and is more significant in turbulent flow conditions. By the time the Reynolds number was around 700 to 5000, The maximum and 
minimum Nusselt numbers obtained for each variation of the tube cross-section are 24,248 and 5,908 for elliptical tube; 19, 673 and 5.276 for triangular tube; 12,409 and 7,456 for square tube; and 11,884 and 6,189 for the circular tube.

\section{CONCLUSIONS}

The convective heat transfer characteristics of the four cross-sectional variations with the flow in the tube in constant heat flux were investigated experimentally. The results show that changes in the geometry of the tube cross-section affect heat transfer performance. The elliptical tube has the greatest heat transfer performance compared to the tube with other crosssections; therefore, the heat transfer in the elliptical tube is more effective. Then sequentially, the triangular tube has a greater heat transfer performance than the square tube, followed by the circular tube. When the Reynolds number is around 700 to 5000, the maximum convective coefficient and Nusselt number are obtained for each variation of the tube cross-section, namely $2069,035 \mathrm{~W} / \mathrm{m}^{2} . \mathrm{K}$ and 24,248 for the elliptical tube; $1699,549 \mathrm{~W} / \mathrm{m}^{2} . \mathrm{K}$ and 19,673 for a triangular tube; $779,099 \mathrm{~W} / \mathrm{m}^{2} . \mathrm{K}$ and 12,409 for square tube; and $588,712 \mathrm{~W} / \mathrm{m}^{2} . \mathrm{K}$ and 11,884 for circular tube. The results also show that the heat transfer performance will increase as the Reynolds number increases in each variation of the tube cross-section. This increase is low under laminar flow conditions and will be clear and significant in a turbulent flow.

\section{ACKNOWLEDGEMENT}

Thanks to Universitas Sriwijaya for the facilities of this research.

\section{REFERENCES}

[1] A. Saleh, S. Rasheed, and R. Smasem, "Convection Heat Transfer in a Channel of Different Cross Section Filled With Porous Media," Kufa J. Eng., vol. 09, no. 02, pp. 57-73, 2018, doi: 10.30572/2018/kje/090205.

[2] S. A. Rasheed and J. M. Abood, "Force Convection Heat transfer from a Different Cross Section Cylinder Embedded in Porous Media," J. Eng. Sci., vol. 20, no. 3, pp. 727-736, 2017, doi: 10.13140/RG.2.2.14800.25600.

[3] K. Sutthivirode and T. Suparos, "A study of forced convection heat transfer : Constant heat flux," Proc. 2018 5th Int. Conf. Bus. Ind. Res. Smart Technol. Next Gener. Information, Eng. Bus. Soc. Sci. ICBIR 2018, pp. 236-241, 2018, doi: 10.1109/ICBIR.2018.8391199.

[4] P. Bisht, M. Joshi, and A. Gupta, "Comparison of Heat Transfer between a Circular and Rectangular Tube Heat Exchanger by using Ansys Fluent," Int. J. Therm. Technol., vol. 4, no. 2, pp. 88-92, 2014.

[5] W. Dang and L. B. Wang, "Convective heat transfer enhancement mechanisms in circular tube inserted with a type of twined coil," Int. J. Heat Mass Transf., vol. 169, p. 120960, 2021, doi: 10.1016/j.ijheatmasstransfer.2021.120960.

[6] S. M. Elsherbiny, M. A. Teamah, and A. R. Moussa, "Experimental mixed convection heat transfer from an isothermal horizontal square cylinder," Exp. Therm. Fluid Sci., vol. 82, pp. 459471, 2017, doi: 10.1016/j.expthermflusci.2016.12.002.

[7] S. Zeinali Heris, F. Oghazian, M. Khademi, and E. Saeedi, "Simulation of Convective Heat Transfer and Pressure Drop in Laminar Flow of Al 2 O 3 /water and CuO/water Nanofluids Through Square and Triangular Cross-Sectional Ducts," J. Renew. Energy Environ., vol. 2, no. 1, pp. 6-18, 2015.

[8] R. Kumar, Varun, and A. Kumar, "Thermal and fluid dynamic characteristics of flow through triangular cross-sectional duct: A review," Renew. Sustain. Energy Rev., vol. 61, pp. 123-140, 2016, doi: 10.1016/j.rser.2016.03.011.

[9] Y. S. Muzychka and M. M. Yovanovich, "Laminar forced convection heat transfer in the combined entry region of non-circular ducts," J. Heat Transfer, vol. 126, no. 1, pp. 54-61, 2004, doi: $10.1115 / 1.1643752$. 
[10] M. Norouzi, M. M. Shahmardan, M. Davoodi, and M. Mahmoudi, "An Exact Solution for Fluid Flow and Heat Convection through Triangular Ducts Considering the Viscous Dissipation," AUT J. Mech. Eng. AUT, vol. 3, no. 2, pp. 197-204, 2019, doi: 10.22060/ajme.2018.14630.5737.

[11] M. Venkateswara Rao, P. V. Ravi Kumar, and P. S. Sankara Rao, "Laminar flow heat transfer in concentric equilateral triangular annular channels," Indian J. Chem. Technol., vol. 13, no. 6, pp. 614-622, 2006.

[12] M. Yang, X. Wang, Z. Wang, Z. Li, and Y. Zhang, "Correlation For Turbulent Convection Heat Transfer In Elliptical Tubes By Numerical Simulations," Front. Heat Mass Transf., vol. 7, no. 11, pp. 1-6, 2018, doi: 10.5098/hmt.11.7.

[13] D. Taler and J. Taler, "Simple heat transfer correlations for turbulent tube flow," E3S Web Conf., vol. 13, pp. 1-7, 2017, doi: 10.1051/e3sconf/20171302008.

[14] S. Kaprawi, P. Dewi,Nukman, "Experimental Study Of Convective Heat Transfer Of Alumina Oxide Nanofluids In Triangle Channel With Uniform Heat Flux," Front. Heat Mass Transf. (FHMT), vol. 22, no. 16, pp. 1-6, 2021, doi: 10.5098/hmt.16.22.

[15] M. M. Janjua, N. U. Khan, W. A. Khan, W. S. Khan, and H. M. Ali, "Numerical study of forced convection heat transfer across a cylinder with various cross sections," J. Therm. Anal. Calorim., vol. 143, no. 3, pp. 2039-2052, 2021, doi: 10.1007/s10973-020-10297-7. 